\title{
Clinical experience and skills of physicians in hospital cardiac arrest teams in Denmark: a nationwide study
}

This article was published in the following Dove Press journal:

Open Access Emergency Medicine

7 March 2017

Number of times this article has been viewed

\author{
Kasper G Lauridsen ${ }^{1-3}$ \\ Anders S Schmidt ${ }^{1-3}$ \\ Philip Caap ${ }^{3,4}$ \\ Rasmus Aagaard ${ }^{2,3,5}$ \\ Bo Løfgren ${ }^{1,3,4}$
}

'Department of Internal Medicine, ${ }^{2}$ Clinical Research Unit, Regional Hospital of Randers, Randers,

${ }^{3}$ Research Center for Emergency Medicine, Aarhus University Hospital, ${ }^{4}$ Institute of Clinical Medicine, Aarhus University, Aarhus, ${ }^{5}$ Department of Anesthesiology, Randers Regional Hospital, Denmark
Correspondence: Bo Løfgren

Department of Internal Medicine, Randers Regional Hospital, Skovlyvej I, 8930 Randers NE, Denmark

Tel +4578420000

Email bl@clin.au.dk
Background: The quality of in-hospital resuscitation is poor and may be affected by the clinical experience and cardiopulmonary resuscitation (CPR) training. This study aimed to investigate the clinical experience, self-perceived skills, CPR training and knowledge of the guidelines on when to abandon resuscitation among physicians of cardiac arrest teams.

Methods: We performed a nationwide cross-sectional study in Denmark. Telephone interviews were conducted with physicians in the cardiac arrest teams in public somatic hospitals using a structured questionnaire.

Results: In total, 93 physicians (53\% male) from 45 hospitals participated in the study. Median age was 34 (interquartile range: 30-39) years. Respondents were medical students working as locum physicians (5\%), physicians in training (79\%) and consultants $(16 \%)$, and the median postgraduate clinical experience was 48 (19-87) months. Most respondents (92\%) felt confident in treating a cardiac arrest, while fewer respondents felt confident in performing intubation (41\%) and focused cardiac ultrasound (39\%) during cardiac arrest. Median time since last CPR training was 4 (2-10) months, and 48\% had attended a European Resuscitation Council (ERC) Advanced Life Support course. The majority (84\%) felt confident in terminating resuscitation; however, only $9 \%$ were able to state the ERC guidelines on when to abandon resuscitation.

Conclusion: Physicians of Danish cardiac arrest teams are often inexperienced and do not feel competent performing important clinical skills during resuscitation. Less than half have attended an ERC Advanced Life Support course, and only very few physicians know the ERC guidelines on when to abandon resuscitation.

Keywords: cardiopulmonary resuscitation, Advanced Cardiac Life Support, graduate medical education, resuscitation orders

\section{Introduction}

The quality of cardiopulmonary resuscitation (CPR) during in-hospital cardiac arrest is poor. ${ }^{1}$ Resuscitation errors including erroneous airway management, delayed defibrillation, lack of vascular access and drug administration often occur during CPR, resulting in decreased survival. ${ }^{2}$ Frequent problems during CPR include airway management and inappropriate staffing, for example, the absence of an anesthetist or intensivist. ${ }^{3}$

Clinical experience and skills are important during resuscitation. In pediatric cardiac arrest, unsuccessful resuscitation is more likely when the primary nurse has $<1$ year of experience. ${ }^{4}$ Furthermore, the presence of an advanced life support (ALS) provider is reported to improve survival after in-hospital cardiac arrest. ${ }^{5}$

The cardiac arrest team composition differs among hospitals. Teams mostly include physicians, nurses and orderlies from different departments. ${ }^{6}$ However, the 
clinical experience, self-perceived resuscitation skills and the CPR training level of the cardiac arrest team members remain unknown.

We aimed to investigate the clinical experience, selfperceived CPR skills, CPR training and knowledge on guidelines on when to abandon resuscitation among physicians in-hospital cardiac arrest teams.

\section{Materials and methods Data collection}

We performed a nationwide study by conducting telephone interviews with physicians on in-hospital cardiac arrest teams. In a recent study, all public somatic hospitals in Denmark $(\mathrm{N}=47)$ and their cardiac arrest team composition were identified. ${ }^{6}$ Physicians serving at private and psychiatric hospitals and hospitals serving outpatients only were excluded. In Denmark, cardiac arrest teams are ad hoc teams composed of hospital personnel on duty, for example, one to three physicians, nurses and orderlies. ${ }^{6}$ Some Danish hospitals have an early response team. However, a cardiac arrest team will be summoned in case of cardiac arrest. All physicians who were members of the cardiac arrest team in each hospital on the day of the telephone interview were contacted. If a physician refused to participate, a new telephone call was made on a subsequent day to the physician covering the same function. If a physician was unable to be interviewed at the time of the telephone call due to clinical duties, a subsequent call was agreed upon. Telephone interviews were conducted using a structured questionnaire by three of the investigators during 1 month in 2014 between 9 am and 9 pm. According to Danish law, no approval from the Danish National Committee on Biomedical Research Ethics was required, as the study did not involve human biological material (Danish Act on Research Ethics, Review of Health Research Projects, Act number 593 of 14 July 2011 section 14 (2)).

\section{Study questionnaire}

The questionnaire was divided into five parts: 1) information on the cardiac arrest team composition and allocation of tasks, including the role of the respondent; 2) demographics including age, sex, area of specialty, postgraduate clinical experience and clinical position; 3) questions regarding the respondents' self-perceived technical skills; 4) information on time since last CPR course and attendance to a European Resuscitation Council (ERC) ALS course and 5) knowledge on when to abandon resuscitation according to the ERC 2010 guidelines. $^{7}$

Responses on self-perceived skill performance during cardiac arrest were collected dichotomously (yes/no). When asked about confidence in treating cardiac arrest, physicians were presented a 5-point Likert scale. ERC 2010 guidelines on when to abandon resuscitation were defined by the statement: "In general, resuscitation should be continued as long as VF persists. It is generally accepted that ongoing asystole for more than $20 \mathrm{~min}$ in the absence of a reversible cause, and with ongoing ALS, constitutes grounds for abandoning further resuscitation attempts". ${ }^{7,8}$ The questionnaire was completed on paper during each telephone interview and subsequently entered into a database.

\section{Data analysis}

All questionnaires were entered into a database twice to correct for typing errors. Data were analyzed for normality using histograms and Q-Q plot analysis. All data were nonparametric and reported as median (interquartile range). Nonparametric data were compared using Wilcoxon rank sum test. Data were analyzed using Stata version 13.0 (StatsCorp LP, College Station, TX, USA).

\section{Results}

In total, 94 physicians were contacted and one refused to participate, which resulted in 93 interviews. The interview was performed during the first telephone call in 52 cases, and 41 interviews were conducted during the subsequent call. The interviews covered 45 hospitals, as two Danish hospitals did not have a physician in the cardiac arrest team.

The median number of physicians in the cardiac arrest teams was $2(1-3)$. Respondents ( $\mathrm{N}=93$, male: $53 \%$ ) included $5(5 \%)$ medical students working as locum physicians, 73 (79\%) physicians in training and 15 (16\%) consultants. Further characteristics of the respondents are presented in Figure 1.

Overall, 49 physicians (53\%) came from Department of Internal Medicine, 13 (14\%) from Department of Cardiology, $30(32 \%)$ from Department of Anesthesiology and one (1\%) from Department of Surgery. Physicians from the Department of Internal Medicine had a median clinical experience of 27 months (interquartile range: 12-60), while physicians from the Department of Anesthesiology had a median clinical experience of 90 months (53-194; $p<0.0001)$. Physicians from the Department of Internal Medicine treated less cardiac arrests with a median of six arrests (2-20) versus 50 arrests (30-100) treated by physicians from the Department of Anesthesiology $(p<0.0001)$.

Most physicians (62\%) had a more senior colleague on call at the hospital, who was not a member of the cardiac arrest team, and $74 \%$ of nonconsultants had a more senior 
A

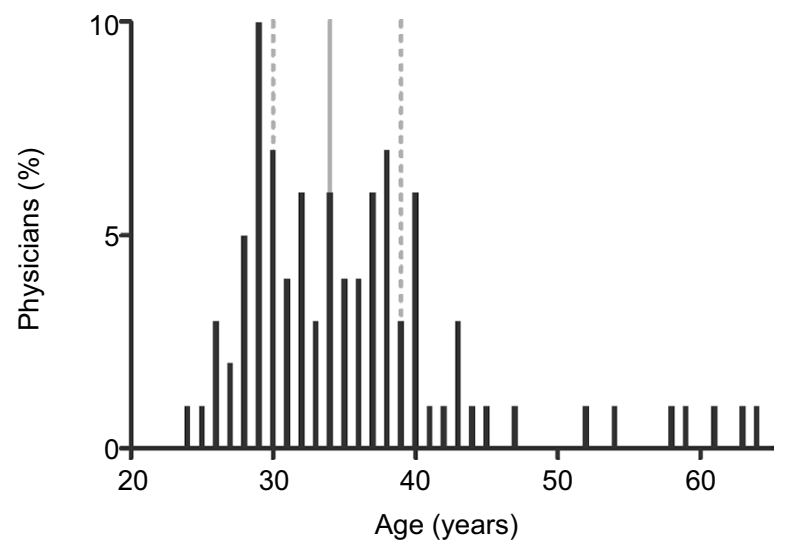

C Total count of cardiac arrests treated per physician

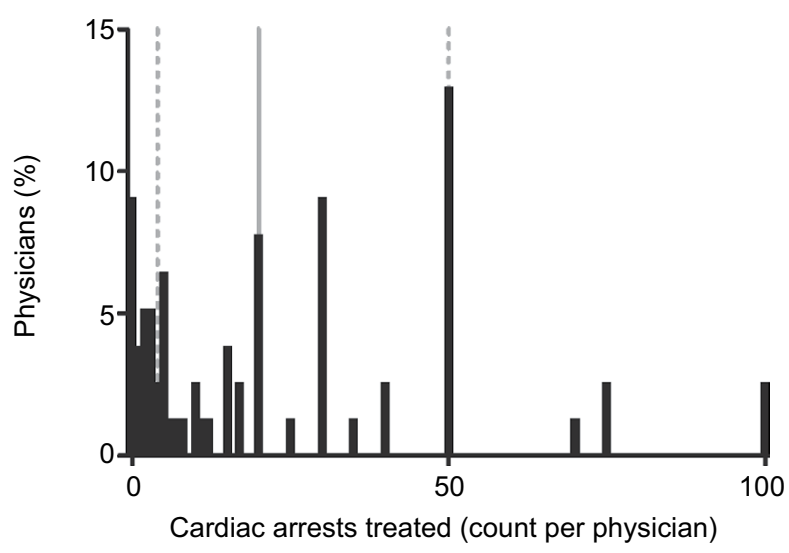

B

Physicians' clinical experience

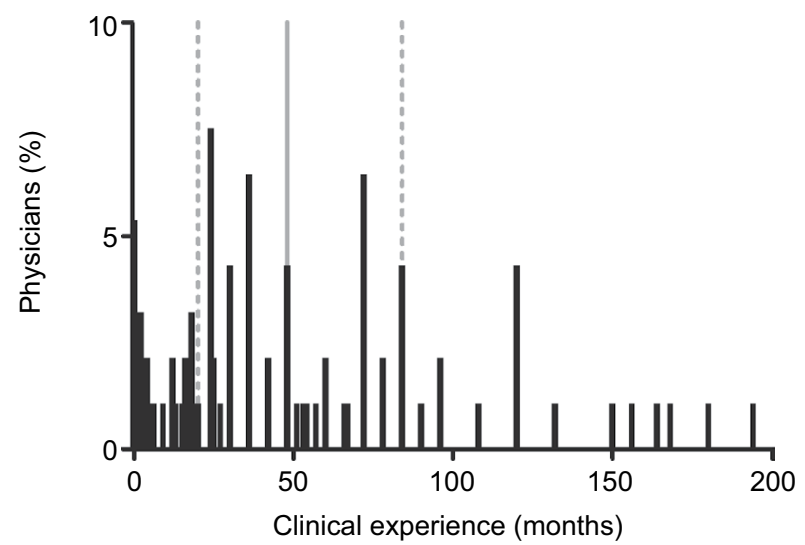

D Physicians' occupation and department

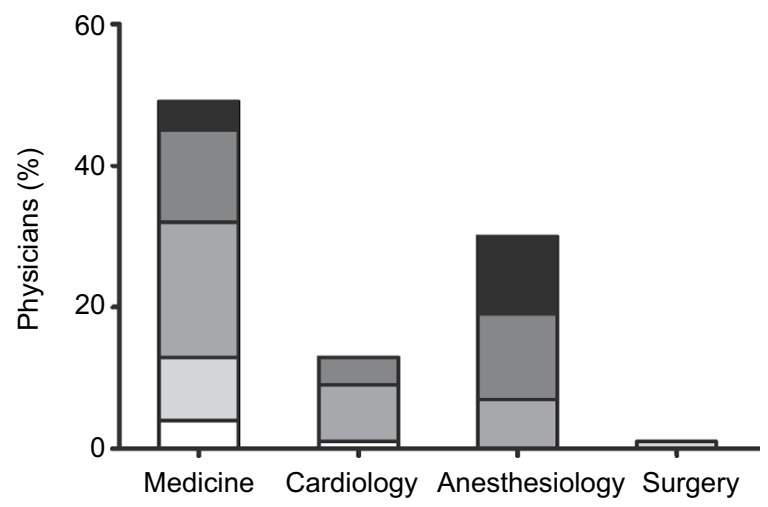

Figure I Characteristics of physicians in cardiac arrest teams $(\mathrm{N}=93)$.

Notes: Full line: median; dashed line: Ist quartile and 3rd quartile. (A) Physicians' age. Median age was 34 (30-39) years. (B) Physicians' clinical experience. Median postgraduate clinical experience was 48 (19-87) months. The clinical experience of six physicians $(336,342,348,360,408$ and 444 months, respectively) is not shown in the figure. (C) Total count of cardiac arrests treated per physician. Median number of cardiac arrests treated was 20 (4-50). Six physicians reported to have treated 200 cardiac arrests and two physicians I,000 cardiac arrests, which are not shown in the figure. (D) Physicians' occupation and department allocation. $\square$ Medical student (medical student working as locum physician; locum physician); $\square$ house officer (physician with up to I year of postgraduate clinical experience); $\square$ senior house officer (physician with >I to $\sim 3$ years of postgraduate clinical experience); $\square$ specialist registrar (physician in specialist training); $\square$ consultant (board-certified specialist).

Table I Physicians' agreement with the statement "I feel confident treating a cardiac arrest" ( $\mathrm{N}=93)$ assessed on a 5-point Likert scale

Physicians self-perceived confidence in treating cardiac arrest

Overall, Department of Department of

n (\%) Internal Medicine, Anesthesiology, n (\%) n (\%)

\begin{tabular}{llll}
\hline Disagree & $0(0)$ & $0(0)$ & $0(0)$ \\
Partially disagree & $2(2)$ & $2(4)$ & $0(0)$ \\
Nor/either & $5(5)$ & $3(6)$ & $1(3)$ \\
Partially agree & $40(43)$ & $27(55)$ & $6(20)$ \\
Agree & $46(49)$ & $17(35)$ & $23(77)$ \\
\hline
\end{tabular}

colleague on call not included in the cardiac arrest team. In total, 86 physicians (92\%) agreed or partially agreed that they felt confident in treating cardiac arrest (Table 1). Physicians from the Department of Anesthesiology felt more confident in treating cardiac arrest, compared with physicians from the Department of Internal Medicine $(p=0.004)$. Table 2 shows physicians' self-evaluated skills. A median of 4 (2-10) months had passed since completion of any CPR training. In total, 45 (48\%) physicians had completed an ERC-certified ALS course.

Overall, $8(9 \%)$ of the physicians were able to state the ERC guidelines on when to abandon resuscitation, 27 (29\%) gave an incorrect answer and $58(62 \%)$ stated that they did not know the guidelines.

\section{Discussion}

We found that physicians in cardiac arrest teams were most often in training with 4 years or less of postgraduate clinical 
Table 2 Perceived cardiopulmonary resuscitation skills among all physician members in the cardiac arrest teams $(\mathrm{N}=93)$

\begin{tabular}{ll}
\hline Clinical skills during resuscitation & $\mathbf{n}(\%)$ \\
\hline Mouth-to-mouth ventilation & $91(98)$ \\
Oro-naso-pharyngeal airway & $84(90)$ \\
Bag-mask ventilation & $93(100)$ \\
Larynx-mask & $56(60)$ \\
Intubation & $38(4 I)$ \\
IV access & $92(99)$ \\
Intraosseous access & $68(73)$ \\
Central vein catheter placement & $34(37)$ \\
Direct current cardioversion & $88(95)$ \\
Transcutaneous pacing & $70(75)$ \\
Focused cardiac ultrasound & $36(39)$ \\
Termination of resuscitation & $78(84)$ \\
\hline
\end{tabular}

Note: Respondents were asked if they felt competent in performing each skill during resuscitation.

Abbreviation: IV, intravenous.

experience. Most physicians felt overall competent in treating cardiac arrest. However, many physicians did not feel confident in performing the clinical skills important for resuscitation. Furthermore, only half of the physicians had ever completed an ERC ALS course. The majority responded that they were able to decide when to terminate resuscitation; however, only a few knew the ERC guidelines on when to abandon resuscitation.

Physicians in cardiac arrest teams are reported to be mainly senior house officers and specialist registrars. ${ }^{6,9}$ However, clinical experience, CPR training and skills of these members have previously not been examined. Interestingly, some of the members $(5 \%)$ of the cardiac arrest team were medical students working as locum physicians.

We found that nonconsultants have more senior colleagues available at the hospital, who are not members of the cardiac arrest team. The background for not including these senior physicians remains unknown. Previous studies suggest that clinical experience is important for resuscitation outcome in the pediatric intensive care unit and in the prehospital setting. ${ }^{4,10}$ Clinical experience may furthermore improve team leadership performance. ${ }^{11}$ Therefore, including more senior physician members may be considered in organization of cardiac arrest teams in future. However, experience in treating cardiac arrest may be more important than clinical seniority alone. According to our data, one-fourth of the physicians have treated four cardiac arrests or less, indicating limited experience. Nonetheless, one-fourth also reported having treated $>50$ cardiac arrests. The optimal clinical experience among cardiac arrest team members is unknown.

The cardiac arrest team members are required to perform a number of tasks during and after resuscitation. The
Resuscitation Council (UK) recommends that the cardiac arrest team should perform a minimum set of skills, that is, mask ventilation, use of a supraglottic airway, defibrillation, intravenous cannulation, drug administration and skills required for immediate post-resuscitation care. ${ }^{12}$ In our study, less than half of the physicians felt competent in performing intubation. However, nurse anesthetists with skills in airway management are included in all cardiac arrest teams in Denmark. ${ }^{6}$ The majority of physicians were unable to perform focused cardiac ultrasound. This is in contrast to international resuscitation guidelines stating that there is no doubt that echocardiography has the potential to identify reversible causes of cardiac arrest. ${ }^{13}$ Furthermore, one-fourth responded that they were not able to perform transcutaneous pacing which is essential in case of complete atrioventricular block in cardiac arrest and in the peri-arrest setting. ${ }^{13}$ Interestingly, the majority of physicians were nonanesthesiologists or noncardiologists, even though anesthesiologists ${ }^{3}$ and cardiologists possess skills that are important for resuscitation. Cardiac arrest teams should be organized to include every clinical skill required during resuscitation and post-arrest.

We found that more than half of the physicians have not completed an ERC ALS course. This is noteworthy since 30-day and 1-year survival are reported to be improved when at least one CPR provider has ALS training. ${ }^{5}$ We found that physicians had a median of 4 months since the last CPR training, which is a shorter retraining interval than found in previous studies. ${ }^{14,15}$ However, CPR performance rapidly declined as soon as after 3-6 months,${ }^{16}$ and therefore, it may be of concern that one-fourth of the physicians had CPR training $>10$ months ago.

In our study, the majority felt competent in deciding when to abandon resuscitation, but only $9 \%$ were able to state the ERC 2010 guideline on when to abandon resuscitation. Furthermore, approximately one-third of the physicians claimed to know the guideline on when to abandon resuscitation, but they were unable to state the guideline correctly. This underlines that ethical issues in resuscitation should be emphasized in future resuscitation courses.

\section{Limitations}

This nationwide study is based on telephone interviews conducted with a limited sample of physicians in cardiac arrest teams. This study investigated only the physicians in the cardiac arrest team. Hence, we cannot infer on other team members, for example, nurses and orderlies. Interviews were performed between 9 am and $9 \mathrm{pm}$, and physician characteristics during nights may differ. We can only infer 
on the physicians' self-perceived skills, and not their actual skill performance. Finally, self-perceived skills and number of cardiac arrests treated may be reported to be lower if collected anonymously, for example, in a written questionnaire.

\section{Conclusion}

Physicians in Danish cardiac arrest teams are often inexperienced and do not feel competent performing important clinical skills during resuscitation. Less than half have attended an ERC ALS course, and only very few physicians know the ERC guidelines on when to abandon resuscitation.

\section{Disclosure}

The authors report no conflicts of interest in this work.

\section{References}

1. Abella BS, Alvarado JP, Myklebust H, et al. Quality of cardiopulmonary resuscitation during in-hospital cardiac arrest. JAMA. 2005; 293(3):305-310.

2. Ornato JP, Peberdy MA, Reid RD, Feeser VR, Dhindsa HS; NRCPR Investigators. Impact of resuscitation system errors on survival from in-hospital cardiac arrest. Resuscitation. 2012;83(1):63-69.

3. Time to intervene? A review on patients who underwent cardiopulmonary resuscitation as a result of an in-hospital cardiorespiratory arrest. Available from: http://www.ncepod.org.uk/2012report1/downloads/ CAP_fullreport.pdf. Accessed November 30, 2014.

4. Gaies MG, Clarke NS, Donohue JE, Gurney JG, Charpie JR, Hirsch JC. Personnel and unit factors impacting outcome after cardiac arrest in a dedicated pediatric cardiac intensive care unit. Pediatr Crit Care Med. 2012;13(5):583-588.
5. Moretti MA, Cesar LA, Nusbacher A, Kern KB, Timerman S, Ramires JA Advanced cardiac life support training improves long-term survival from in-hospital cardiac arrest. Resuscitation. 2007;72(3):458-465.

6. Lauridsen KG, Schmidt AS, Adelborg K, Lofgren B. Organisation of inhospital cardiac arrest teams - a nationwide study. Resuscitation. 2015; 89:123-128

7. Lippert FK, Raffay V, Georgiou M, Steen PA, Bossaert L. European Resuscitation Council Guidelines for Resuscitation 2010 Section 10. The ethics of resuscitation and end-of-life decisions. Resuscitation. 2010; 81(10):1445-1451.

8. Bonnin MJ, Pepe PE, Kimball KT, Clark PS Jr. Distinct criteria for termination of resuscitation in the out-of-hospital setting. JAMA. 1993; 270(12):1457-1462.

9. Pittman J, Turner B, Gabbott DA. Communication between members of the cardiac arrest team - a postal survey. Resuscitation. 2001; 49(2): 175-177.

10. Soo LH, Gray D, Young T, Skene A, Hampton JR. Influence of ambulance crew's length of experience on the outcome of out-of-hospital cardiac arrest. Eur Heart J. 1999;20(7):535-540.

11. Cooper S, Wakelam A. Leadership of resuscitation teams: 'Lighthouse Leadership'. Resuscitation. 1999;42(1):27-45.

12. Quality standards for cardiopulmonary resuscitation practice and training from the Resuscitation Council (UK). Available from: http://www. resus.org.uk/pages/QSCPR_Acute.pdf. Accessed June 5, 2014.

13. Soar J, Nolan JP, Bottiger BW, et al. European Resuscitation Council Guidelines for Resuscitation 2015: Section 3. Adult advanced life support. Resuscitation. 2015;95:100-147.

14. Siebig S, Kues S, Klebl F, et al. Cardiac arrest: composition of resuscitation teams and training techniques: results of a hospital survey in German-speaking countries. Dtsch Arztebl Int. 2009;106(5):65-70.

15. Schmidt AS, Lauridsen KG, Adelborg K, Lofgren B. Hospital implementation of resuscitation guidelines and review of CPR training programmes: a nationwide study. Eur J Emerg Med. 2016;23(3): 232-234.

16. Smith KK, Gilcreast D, Pierce K. Evaluation of staff's retention of ACLS and BLS skills. Resuscitation. 2008;78(1):59-65.
Open Access Emergency Medicine

\section{Publish your work in this journal}

The Open Access Emergency Medicine is an international, peerreviewed, open access journal publishing original research, reports, editorials, reviews and commentaries on all aspects of emergency medicine. The manuscript management system is completely online and includes a very quick and fair peer-review system, which is all

\section{Dovepress}

easy to use. Visit http://www.dovepress.com/testimonials.php to read real quotes from published authors. 